\title{
Multi-spectral Image Analysis for Astaxanthin Coating Classification
}

\author{
Ljungqvist, Martin Georg; Ersbøll, Bjarne Kjær; Nielsen, Michael Engelbrecht; Frosch, Stina
}

Published in:

Scandinavian Workshop on Imaging Food Quality 2011

Publication date:

2011

Document Version

Publisher's PDF, also known as Version of record

Link back to DTU Orbit

Citation (APA):

Ljungqvist, M. G., Ersbøll, B. K., Nielsen, M. E., \& Frosch, S. (2011). Multi-spectral Image Analysis for Astaxanthin Coating Classification. In Scandinavian Workshop on Imaging Food Quality 2011: Ystad, May 27, 2011 - Proceedings (pp. 63-68). Technical University of Denmark. D T U Compute. Technical Report No. 2011. 15 http://www2.imm.dtu.dk/projects/SWIFQ/

\section{General rights}

Copyright and moral rights for the publications made accessible in the public portal are retained by the authors and/or other copyright owners and it is a condition of accessing publications that users recognise and abide by the legal requirements associated with these rights.

- Users may download and print one copy of any publication from the public portal for the purpose of private study or research.

- You may not further distribute the material or use it for any profit-making activity or commercial gain

- You may freely distribute the URL identifying the publication in the public portal 


\title{
Multi-spectral Image Analysis for Astaxanthin Coating Classification
}

\author{
Martin Georg Ljungqvist ${ }^{1,2}{ }^{\star}$ Bjarne Kjær Ersbøll ${ }^{1}$, Michael Engelbrecht \\ Nielsen ${ }^{2}$, Stina Frosch ${ }^{2}$ \\ 1. Technical University of Denmark (DTU), \\ Department of Informatics and Mathematical Modelling \\ 2. Technical University of Denmark (DTU), \\ National Food Institute, Division of Industrial Food Technology
}

\begin{abstract}
Industrial quality inspection using image analysis on astaxanthin coating in aquaculture feed pellets is of great importance for automatic production control. In this study multi-spectral image analysis of pellets was performed using LDA, QDA, SNV and PCA on pixel level and mean value of pixels for each pellet. Classification using LDA or QDA on pellet mean or median values showed better results than using the pixel values or PCA.
\end{abstract}

Keywords: astaxanthin, multi-spectral, image analysis

\section{Introduction}

Industrial quality inspection using image analysis is an area of extensive development. Pigment inclusion in aquaculture feed pellets is of great interest for automatic visual analysis for statistical production control and optimisation.

Astaxanthin is a naturally occurring carotenoide with a high antioxidant activity essential for reproduction, growth and survival, and important for the development of colour in salmonide fishes [1]. The primary use of astaxanthin within aquaculture is as a feed additive to ensure that farmed salmon and trout have similar appearance as their wild counterparts [2]; it is the pigment that makes salmonide fishes red. The colour appearance of fish products is important for the customers. Astaxanthin is highly expensive [3] and therefore optimisation of its use in fish feed production is of importance.

An automatic vision system for on-line quality control of pigment inclusion will be of great benefit to the industry both in relation to process control and process optimisation.

This paper is based in part on an earlier study by Ljungqvist et al. (2010) [4]. Besides this no further work has to the authors' knowledge previously been done on analysing the coating of fish feed using image analysis. Multi-spectral image analysis has shown good results in previous biological applications $[5,6,7,8]$ where it is of interest to detect subtle differences in colour and surface chemistry.

\footnotetext{
`malj@imm.dtu.dk
} 
The aim of this project is to investigate the possibility of distinguishing between feed pellets coated with fish oil with and without added astaxanthin using multi-spectral image analysis and in this way investigate what spectral features are of interest for further analysis of astaxanthin coating.

\section{Material and Methods}

\subsection{Material}

The feed type used is EcoLife20 and AquaLife R90, both with the radius of 4.5 $\mathrm{mm}$. The fish feed pellets are divided into two groups. One class constitutes pellets coated with fish oil with $50 \mathrm{ppm}$ added of a synthetic version of astaxanthin; class A (astaxanthin). The other class is the same pellet types with fish oil coated without additional astaxanthin included; class B (base). (The fish oil typically contains a small amount of natural astaxanthin, but this is assumed to be less than 1 ppm and should therefore not affect the results.) The distribution of the surface coating is unknown and some amount of variation is likely to occur.

A total of 2223 EcoLife20 pellets were used, and a total of 2158 AquaLife R90 pellets were used, see Table 1.

\subsection{Imaging Equipment}

The equipment used was a camera and lighting system called VideometerLab which supports a multi-spectral resolution of up to 20 wavelengths. These are distributed over the ultra-violet A (UVA), visible (VIS) and first near infrared (NIR) region. The range is from 385 to $1050 \mathrm{~nm}$.

This system uses a Point Grey Scorpion SCOR-20SOM grey-scale camera and the objects of interest are placed inside an integrating sphere (Ulbricht sphere) with uniform diffuse lighting from light emitting diodes (LED) placed around the rim of the sphere. The curvature of the sphere and its white matte coating ensures a uniform diffuse light so that specular effects are avoided and likewise minimising the amount of shadows. The device is calibrated radiometrically with a following light and exposure calibration. The system is geometrically calibrated to ensure pixel correspondence for all spectral bands [9].

The image resolution is $1280 \times 960$ pixels. Each file contains 20 images, one for each spectral band. This results in a multi-spectral image cube with dimensions of $1280 \times 960 \times 20$.

Table 1. Number of analysed pellets in each group.

\begin{tabular}{lcccc} 
& $\begin{array}{c}\text { Size } \\
(\mathrm{mm})\end{array}$ & $\begin{array}{c}\text { Class A } \\
\text { samples }\end{array}$ & $\begin{array}{c}\text { Class B } \\
\text { samples }\end{array}$ & $\begin{array}{c}\text { Total } \\
\text { samples }\end{array}$ \\
\hline EcoLife & 4.5 & 1165 & 1058 & 2223 \\
R90 & 4.5 & 1207 & 951 & 2158 \\
\hline
\end{tabular}




\subsection{Spectral Equipment}

In order to further explore the spectral properties of astaxanthin a spectrometer was used. Absorption spectra of synthetic astaxanthin in a solution of fish oil along with plain fish oil were recorded in the VIS and NIR range using a NIRSystems 6500 absorption spectrometer. The absorption spectra was transformed to reflection values using the standard relation $A=-\log (R)$, where $A$ is absorption values and $R$ is the reflection values.

\subsection{Image Analysis}

The pellets were segmented from the background using a grey-scale threshold.

The basic pellet compound gives a spectral response which will be present in both class A and B. Each pixel is thus a combination of the reflectance of a set of constituents. This mix is assumed to be of equal amount for each pellet type except for the difference of the astaxanthin coating that we want to isolate in our classification.

The ground truth is that we know that certain pellets are coated with synthetic astaxanthin, but since the surface distribution is unknown it is unclear how much synthetic astaxanthin each of those pixels contains. This gives us an uncertain one-to-many relationship situation.

A way to solve this uncertainty is to represent each pellet using the mean or median of all pixels in a pellet as sample values. In this manner we even out the variance of all pixels in a pellet and each pellet becomes a distinct observation.

In addition to the pellet pixel mean and median values further summary statistics features to describe the coating distribution were extracted based on pellet pixel values: Skewness, kurtosis, variance and maximum value.

PCA Our multivariate data from the images was analysed using principal component analysis (PCA) for exploratory purpose. PCA is the most optimal method with respect to maximising the variance [10] and has been commonly used for dimension reduction for dealing with ill-posed problems. If the relation of interest contains large variation then PCA is a good method for analysing the data.

The pre-processing method standard normal variate (SNV) [11] was used to reduce any variation in concentration level of the overall coating concentration between pellets.

Discriminant Analysis To discriminate between the two classes we want the within group deviation to be small compared to that between groups. Wilk's $\Lambda$ consists in principle of the ratio of the within group variation $(\mathbf{W})$ and the total variation $(\mathbf{T})$, i.e. the within group plus the between group variation. $\Lambda=\frac{\operatorname{det}(\mathbf{W})}{\operatorname{det}(\mathbf{T})}$. A value of Wilk's $\Lambda$ which is close to zero indicates that the two groups are well separated.

For statistical discriminant analysis methods we use linear discriminant analysis (LDA) and quadratic discriminant analysis (QDA) [10]. They are both based 
on the Mahalanobis distance, and assumes that the variables in each class are normally distributed. LDA and QDA are based on a distance to the class mean weighted by the variance. A training set of $70 \%$ of the samples were used here, along with a test set of $30 \%$ of the samples.

\section{Results and Discussion}

It turned out that spectral band number 20 showed some artefacts for about half of the EcoLife20 class A due to temperature variations so therefore statistical tests were also performed without this band. Results show that this problem did not affect the classification in a negative manner (results not shown).

Comparing the SNV-normalised mean spectra of the two classes of EcoLife20 elucidates the largest difference being at 970, 950 and $565 \mathrm{~nm}$ (in order of magnitude). Both 970 and $950 \mathrm{~nm}$ are in the NIR range, while $565 \mathrm{~nm}$ represents the green colour which is next to yellow. For AquaLife R90 the largest difference between the class spectra are in the visual range around $400 \mathrm{~nm}$ and also slightly above $600 \mathrm{~nm}$.

The spectrometer results show a large deviation between synthetic astaxanthin in fish oil and plain fish oil to be in the range of $500-600 \mathrm{~nm}$, see Figure 1. This corresponds well with the results from the VideometerLab images and partly corresponds with previous studies of astaxanthin [12, 13].

The mean spectra of the two groups of both EcoLife20 and AquaLife R90 are significantly different at a $0.1 \%$ level. This is promising for classification between the two coating groups. On the other hand, Wilk's lambda of the class means of EcoLife20 pellet mean values equals 0.987, and for AquaLife R90 it is 0.826 . The high values here are reflecting the situation of high variation within the groups and a low variation between the groups. So even though the class means are well separated, there is a vast overlap of the two groups.

Classification tests of EcoLife20 show that LDA on the pellet means or pellet medians gave the best result with a classification correctness of about $93 \%$. See Table 2 for test results.

Classification tests of AquaLife R90 show that QDA on the pellet medians gave the best result with a classification correctness of $100 \%$.

Using LDA and QDA on the other summary statistics features (skewness, kurtosis, variance and maximum value) gave results of lower correctness for both pellet types (results not shown).

Using PCA before doing LDA or QDA on the pellet mean values did not improve the results, see Table 2. This may be an indication that maximising the variance is not a well-suited method for this particular problem, which also was indicated by the high variation within groups in comparison to the variation between groups. PC2 shows the largest difference between the two classes, see Figure 2. The first five principal components explain $98 \%$ of the total variance of the pellet mean values, and still the result of the discriminant analysis on these five components rendered worse classification in comparison to using the plain data itself. 
Table 2. The misclassification of pellet coating type for different kinds of features. Displayed values are total test error for classification of the two groups A (astaxanthin) and B (base).

\begin{tabular}{lll} 
EcoLife20 & LDA & QDA \\
\hline Mean & 0.0646 & 0.0901 \\
Median & 0.0736 & 0.0931 \\
Mean, SNV, PC1-5 & 0,1396 & 0,2162 \\
\hline AquaLife R90 & & \\
\hline Mean & 0.0046 & 0.0031 \\
Median & 0.0015 & 0.0000 \\
Mean, SNV, PC1-5 & 0.0185 & 0.0201 \\
\hline
\end{tabular}

To sum up, the results show that it is possible to distinguish between feed pellets with and without inclusion of synthetic astaxanthin in the coating using multi-spectral image analysis. However, more work is needed in order to make the method robust for various pellet types and also for various amount of astaxanthin. Since astaxanthin is expensive it is desired to have a good accuracy in the method. This will further on be of importance for developing on-line quality food and feed products with optimal use of pigment and minimum amount of waste.

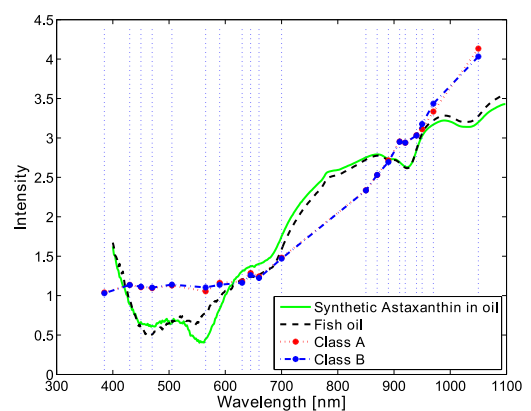

Fig. 1. Spectrometer reflectance of synthetic astaxanthin in oil (green) and plain fish oil (black). Multi-spectral images (reflectance) mean of class A (synthetic astaxanthin in fish oil) (red) and class B (fish oil) (blue) of the EcoLife20 type.
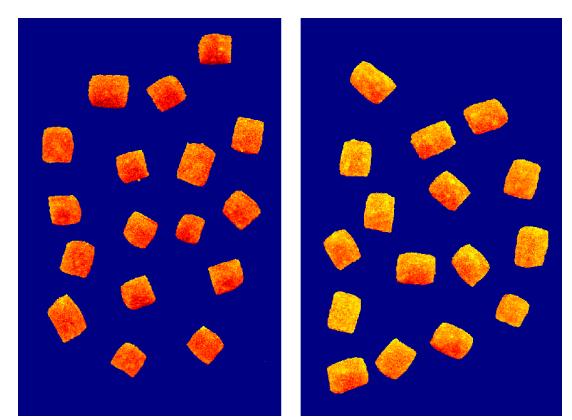

Fig. 2. The 2nd principal component of the multi-spectral image (reflectance) of EcoLife20 pellet pixels. Pellets coated with synthetic astaxanthin in fish oil, class A (left). Pellets coated with fish oil, class B (right). Red colour indicates high values. 


\section{Acknowledgments}

The work presented has received funding from BioMar A/S and the EU under the Seventh Framework Programme FP7/2007-2013 under grant agreement number 214505.10 .

\section{References}

[1] J.B. Owen. Genetic variation and nutrition - edited by a. p. simopoulus and b. childs. Clinical Nutrition, 10(1):61-62, 1991.

[2] O.J. Torrisen, R.W. Hardy, and K.D. Shearer. Pigmentation of salmonids carotenoid deposition and metabolism. Reviews in Aquatic Sciences, 1(2):209 225, 1989.

[3] R.T.M. Baker, A.-M. Pfeiffer, F.-J. Schöner, and L. Smith-Lemmon. Pigmenting efficacy of astaxanthin and canthaxanthin in fresh-water reared atlantic salmon, salmo salar. Animal Feed Science and Technology, 99(1-4):97-106, 2002.

[4] Martin Georg Ljungqvist, Stina Frosch, Michael Engelbrecht Nielsen, and Bjarne K. Ersbøll. Analysis of astaxanthin in fish feed pellets. Proc. West European Fish Technologists Association, 40:59-60, Oct 2010.

[5] David Delgado Gomez, Line Harder Clemmensen, Bjarne K. Ersbøll, and Jens Michael Carstensen. Precise acquisition and unsupervised segmentation of multi-spectral images. Computer Vision and Image Understanding, 106(2-3):183193, 2007.

[6] Line Katrine Harder Clemmensen and Bjarne Kjær Ersbøll. Multispectral recordings and analysis of psoriasis lesions. MICCAI 06 - Workshop on Biophotonics Imaging for Diagnostics and Treatment, October 6, 2006 proceedings, 9th MICCAI Conference, 2006.

[7] Line H. Clemmensen, Michael E. Hansen, Jens C. Frisvad, and Bjarne K. Ersbøll. A method for comparison of growth media in objective identification of penicillium based on multi-spectral imaging. Journal of Microbiological Methods, 69(2):249 $255,2007$.

[8] Bjørn Skovlund Dissing, Line Katrine Harder Clemmensen, Bjarne Kjær Ersbøll, Hanne Løje, and Jens Adler-Nissen. Temporal reflectance changes in vegetables. 2009 IEEE 12th International Conference on Computer Vision Workshops (ICCV Workshops), pages 1917-1922, 2009.

[9] Jørgen Folm-Hansen. On chromatic and geometrical calibration. PhD thesis, Technical University of Denmark, 1999.

[10] Trevor Hastie, Robert Tibshirani, and Jerome Friedman. The Elements of Statistical Learning: Data Mining, Inference, and Prediction. Springer, 2nd edition, February 2009.

[11] Asmund Rinnan, Frans van den Berg, and Søren Balling Engelsen. Review of the most common pre-processing techniques for near-infrared spectra. TrAC Trends in Analytical Chemistry, 28(10):1201-1222, 2009.

[12] Manuel. Buchwald and William P. Jencks. Optical properties of astaxanthin solutions and aggregates. Biochemistry, 7(2):834-843, 1968.

[13] Jian-Ping Yuan and Feng Chen. Identification of astaxanthin isomers in haematococcus lacustris by hplc-photodiode array detection. Biotechnology Techniques, 11(7):455-459, 1997. 